\title{
Health Programs are Social Programs: Navigating Difficult Healthcare Policy Decisions
}

\author{
Samuel Seshadri ${ }^{1}$ \\ samuelseshadri@uvic.ca
}

\begin{abstract}
Apart from public health and preventive medicine campaigns, a health authority funds healthcare programs primarily for the purpose of immediately improving clinical patient outcomes. For individual health treatments, funding decisions by Canadian provincial governments incorporate some equivalent of a cost-benefit calculation, such as the cost-effectiveness analysis (CEA). This research is important to health policy makers because it considers the effects of expanding a CEA to analyze societal impacts that are already of importance to the government when the appropriateness or accuracy of the cost-benefit calculation is unclear. I use the example of in vitro fertilization funding programs to demonstrate the argument that health programs may also address other relevant issues related to the social determinants of health.
\end{abstract}

Keywords: cost-effectiveness analysis; societal perspectives; healthcare decision making; health economics; quality adjusted life year

${ }^{1}$ I would like to acknowledge the support of Dr. Chris Willmore who generously mentored me during this project. 


\section{A Brief Introduction to Health as an Economic Good}

Economists think of health as a tangible good that can be consumed and invested in. That is, the decisions that people make to improve their health (exercising, diet, etc.) are rational because the investment in health allows the individual to consume health by "feeling healthier" in the long run. Healthcare is thought of as solely a consumption good-when an individual consumes it, there is some inherent value attached to the procedure, medication, or treatment. Individuals always have some desire for health, and they must weigh the potential benefits against the potential costs of attaining health in every choice that they make.

Health behaviours have economic externalities associated with them. Externalities in health are costs and benefits that are not incorporated into the underlying price of the action. For example, when someone decides to begin regularly exercising to increase their well-being, they may end up becoming more productive at work or recruiting a friend to exercise with them, therefore creating benefits not associated with the immediate decision to exercise. Medical ailments too can have negative external effects where the loss in health can cause people to lose their jobs or social supports. One way to identify some health externalities is to look at the social determinants of health (see Figure 1). The social determinants represent some areas of life that can be affected by the diagnosis and treatment of a health condition.

The issue is that health and healthcare are expensive- both in monetary and non-monetary terms. Health and healthcare are both considered "normal" economic goods: normal in the sense that the demand for them tends to increase with income. The positive relationship between increased income, increased health, and increased healthcare service demand has been well studied by health economists (Deaton, 2002; Ettner, 1996; Papazoglou \& Galariotis, 2020). Therefore, those with higher incomes are more able to access the tools to acquire health. As a social right, countries around the world (including Canada) have created healthcare systems that increase accessibility to healthcare by spreading the cost of healthcare across the entire population. Much like an individual investing in their health, the publicly funded healthcare payer must now decide between funding or not funding a health treatment or program.

\section{Figure 1}

Quality Adjusted Life Year (QALY) Benefits, Self-Reported Quality of Life, and the Canadian Social Determinants of Health (Canada). Figure created by Samuel Seshadri
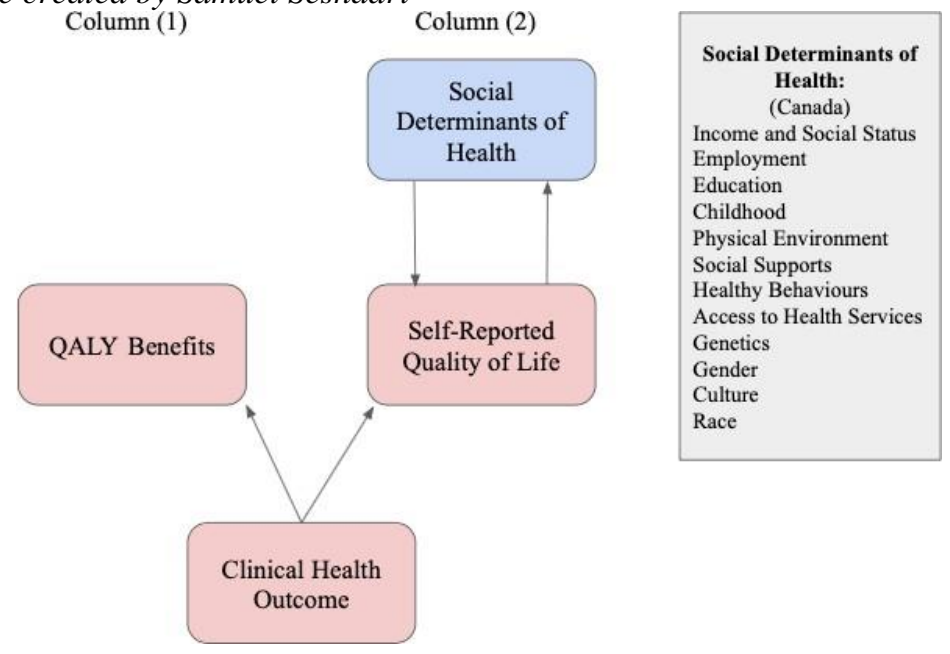


\section{Decisions of the Publicly Funded Healthcare Payer}

In the largely publicly funded Canadian healthcare systems, it is typically the provincial governments who make the decision of whether or not to fund a particular health treatment. Provinces are held accountable by the Canada Health Act-a legal criterion that outlines the standard of health care and ensures coverage "for the purpose of maintaining health, preventing disease or diagnosing injury" (Canada Health Act, 1985, s.2). This coverage is comprehensive, yet it does not include treatments that are not deemed "medically necessary." Governments must find a way to decide which additional programs they will fund.

One way to evaluate health programs is the cost-effectiveness analysis (CEA), which weighs the explicit costs (measured in dollars) of providing a health treatment against a measure of effectiveness. The measurement of effectiveness always focuses on a clinical outcome-such as lives saved, success rate, or percentage improvement from baseline. The governing health authority can use a CEA as a quantitative component of a policy decision. In trauma events or life-threatening conditions (such as a brain tumour or a car accident), a health professional does not need to conduct a CEA - they simply know that the immediate benefit of treating the patient is worth the cost (Orr $\&$ Wolff, 2015). In less severe conditions, however, the choice may not always be so clear. At this point, jurisdictions may start to flip between different funding programs because they are unsure which ones are appropriate. I argue that this issue may be solved by measuring the effects of health programs in non-health aspects of society. Understanding how a health program can also affect the well-being of people in other ways may prompt the funding decision to go in a more socially optimal direction.

Two additional components of the cost-effectiveness analysis are the perspective and the modelling of benefits. First, the perspective of a CEA governs which costs and benefits are to be calculated. The traditional perspective of government assessments is the "publicly funded healthcare payer" which measures explicit treatment costs to the public system and benefits felt by the patient being treated (Canadian Agency for Drugs and Technology in Health, 2017). When researchers need to compare two vastly different treatments or when other clinical effectiveness measures do not exist, Quality Adjusted Life Year (QALY) are used to model benefits. QALY are health utility values anchored between zero and one, where zero represents someone who is dead, and one represents someone of perfect health. The QALY measures the utility of a health condition as a proportion of full health utility. ${ }^{2}$

Literature criticizing QALY benefits for inaccurately representing the true self-reported change in quality of life is not uncommon (Erickson \& Winkelmayer, 2010; Clayton \& MacKay, 2018). QALY weights are primarily calculated from survey data whose participants are precisely chosen. Survey participants vary between those with the health condition, to health professionals, or to those who have not had the condition (Whitehead \& Ali, 2010). The careful selection of survey participants results in QALY gained from treatment failing to be an exact measurement of how much "better" a patient feels post-treatment. However, QALY are the current standard for measuring health benefits.

2 The World Health Organization regularly publishes the Global Health Estimates and the Global Burden of Disease studies that estimate losses in QALY (called disability weights) of a specific health condition (WHO, 2017). Minor health ailments (such as the flu or a temporary broken limb) have QALY values of about 0.8 whereas major ailments (terminal diseases) have QALY values closer to 0.4. 


\section{When QALY Are Not Enough}

The majority of western healthcare systems do not need QALY measurements for evaluation of all healthcare interventions. QALY is rightfully neglected in the case of medically necessary treatments (such as surgery for a brain tumour or stitches for a deep wound). In Canada, the Canada Health Act requires that all medically necessary physician and hospital services are covered. The practice means that care is not given because it is cost-effective, but because it is care that ought to be given. The use of QALY only becomes important when deciding between a selection of treatments, or when the treatment is not clearly a medical necessity. At this point, cost-effectiveness analysis can be applied to investigate the appropriateness of an intervention based on its relative price per QALY gained.

Sometimes a cost-effectiveness ratio is inconclusive, and the health program in question is on the border between cost-effective and not cost-effective. In this case, health authorities may be left in a state of ambivalence toward the treatment. At this point, it is best to expand the view from health programs being a device for immediate health benefits to the view of health programs affecting social welfare.

The idea of a social welfare perspective as a component of a health technology assessment is not new. Organizations such as the Canadian Agency for Drugs and Technology in Health (CADTH) who release guidelines for health technology assessment ask that a societal perspective be taken as an additional viewpoint. Common CEA guidelines include social services, education, and productivity losses (CADTH, 2017). Kim et al. (2020), however, found that the majority of CEAs from the Global Health CEA Registry did not include an appropriate perspective with the most common costs included being productivity (12\%) and transportation costs (21\%). The lack of information inclusion in CEAs may be due to the inherent complexity in the most relevant societal treatment effects or due to researcher ambivalence.

One possible solution to the issue of choosing suitable societal effects is to look at the social determinants of health. The determinants represent areas of society that directly impact the health of citizens. The overall effect of an individual treatment therefore depends on the combination of health services and other factors (See Figure 1). The determinants of health are the first place for researchers to start when establishing potential societal components of an analysis. The difference between QALY benefit measurement and true health benefit (often measured as self-reported quality of life) can be significant.

Figure 1 can be used to make the distinction between QALY measured benefits and benefits that incorporate the broader perspective. Column (1) represents a traditional method of measuring clinical health benefits from successful treatment as QALY. Column (2), however, represents the overall treatment effect on a patient's life as a function of the social determinants of health. Therefore, those health programs that fund treatments that have measurable effects on the social determinants of health have additional evidence beyond QALY benefits.

By expanding analysis to look at the relationship between immediate patient benefits and their sociological determinants one can view a health program as an additional social program that addresses systemic social gaps. The following example of in vitro fertilization (IVF) funding in Canada demonstrates the intersectionality between health programs and social programs. 


\section{An Example: In Vitro Fertilization in Canada}

Infertility is known to affect one in six Canadian couples and has been growing in prevalence due to increases in maternal age (Bushnik et al., 2012; Government of Canada, 2020). IVF is a treatment for infertility that costs roughly $\$ 10,000$ per cycle with a $20-30 \%$ success rate depending on maternal age (Canadian Fertility and Andrology Society, 2019). Public funding of IVF in Canada is available at a limited capacity in Québec, Ontario, Manitoba, and New Brunswick (Mahboob, 2020). Québec has altered IVF public funding decisions twice since 2010, and in November 2020 introduced Bill 73, another potential change to policy that offers one funded cycle of IVF for women between 18 and 40 (National Assembly of Québec, 2020).

From the perspective of IVF as only a health program, QALY gained are the immediate benefits from successfully being treated for infertility. Of course, a successful treatment does not just mean that infertility disappears; it means that a new child has been brought into the world. The child now affects parental quality of life in almost every dimension of the social determinants of health. ${ }^{3}$ Some of the effects have been precisely measured. For example, Lundborg et al. (2017) found that there is a $11-12 \%$ decrease in long-term maternal income as a result of having a child. Therefore, the benefits from having a child through IVF programs are at the expense of lost incomes for those who otherwise would not have been able to conceive.

IVF, however, may also work as a social program for minimizing lost income for an even larger portion of the population- those who are not (yet) infertile. That is, IVF could act as "insurance" of fertility for those who are struggling to decide when the right time for conception is due to competing interests in the workforce. Should IVF be used in this way, potential parents may postpone their pregnancies further because they are covered with IVF in the future and therefore limit losses of income in the labour market.

The above mentioned conclusion is a simple example of how health treatment programs can address the income, employment, and gender social determinants of health. IVF funding in effect may address gender wage gaps, decrease income losses from conception, and therefore alter employment patterns. All three of these issues are already important to governments. For example, in November 2020, the Government of Canada issued new pay equity regulations that update existing regulations for the gender wage gap (2020a). All three of the above issues are systemic in nature and are even greater topics of political discussion than infertility treatments. It therefore may be of government interest to fund IVF because of its dual effects as a contributor to both health and social benefits.

\section{Discussion}

An appropriate counter-argument to cost-effectiveness from the societal perspective is that it is trivial to justify any health program by excessive inclusion of downstream benefits. This is true if looking at health treatment effects on all social determinants of health. For example, to measure every available cost and benefit of child-bearing, the evaluator will soon see that each metric is infinite. If it is costly for a parent to raise a child, then it is costly for the child to ride the school bus which in turn places costs on the bus driver and the children riding the bus, who pass the costs on

\footnotetext{
${ }^{3}$ Other than perhaps genetics from Figure 1.
} 
and on. The same is true for benefits - if a drowning child is saved, there will be benefits from parents, grandparents, and siblings, which increases benefits for other relationships and so on (not to mention that a saved child will pay income tax in the future).

Measurement of every downstream cost and benefit on every social determinant is neither possible nor worthwhile. The effects that are already important to governments are the most useful, and these are the measurements that should be used. In this way, measurements will already have been studied by other government departments and both groups can benefit from learning about potential interdisciplinary effects of policy.

The inclusion of the societal perspective may also disprove the benefits of some health or social policy. If perhaps a proposed policy offered treatment to only those at the bottom quartile of incomes, a U-shaped distribution of healthcare access may form, where the poorest get treatment funded and the wealthiest are covered with health insurance while the middle $50 \%$ of incomes are left to pay out of pocket. This example suggests that although more health coverage can seem beneficial to health, it may also counteract another government policy that targets income inequalities.

Thinking of health programs as social programs also introduces new potential areas of research. The impact of treatment effects on racial, cultural, and gender disparities is as important as it has ever been. Further research into treatment effects on the aging, mental health, and equality in access to care are increasingly relevant. At this level, health programs are not thought of as government payments to make an individual feel better; they are programs that can have far-reaching, mutually beneficial impacts on societal issues at large.

\section{Conclusion}

Overall, the inclusion of a societal perspective in the traditional cost-effectiveness analysis is a recognition that health programs do not exist to promote health within a vacuum. For those programs that do not have a clear yes or no answer, the societal perspective should include the analysis of other non-health issues that are currently important to social welfare. With this knowledge, health programs can potentially be used to promote fairness and address inequality in relation to the social determinants of health. 


\section{References}

Bushnik, T., Cook, J., Yuzpe, A., \& Tough, S. (2012). Estimating the prevalence of infertility in Canada. Human Reproduction, 27, 738-46.

Canadian Agency for Drugs and Technologies in Health. (2017). Guidelines for the economic evaluation of health technologies: Canada. https://www.cadth.ca/about-cadth/how-wedo-it/methods-and-guidelines/guidelines-for-the-economic-evaluation-of-healthtechnologies-canada

Canadian Fertility and Andrology Society. (2019, Sept. 23). Press release. https://cfas.ca/_Library/CARTR/2019_CARTR_Press_Release_PDF.pdf

Clayton, P.A. \& MacKay, D. P.(2018). Experienced utility or decision utility for QALY calculation? Both. Public Health Ethics, 11(1). 82-89.

Deaton, A. (2002). Policy implications of the gradient of health and wealth. Health Affairs, 21(2). 13-30.

Erickson, K. F. \& Winkelmayer, W. C. (2010). The challenges of cost-effectiveness analyses for the clinician. American Journal of Kidney Diseases: The Official Journal of the National Kidney Foundation, 56(6).1023.

Ettner, S. L. (1996). New evidence on the relationship between income and health. Journal of Health Economics, 15(1). 67-85.

Government of Canada. (n.d.). Social determinants of health and health inequalities. https://www. canada.ca/en/public-health/services/health-promotion/population-health/ what-determines-health.html

Government of Canada. (1985). Canada health act. Justice Laws Website. https://lawslois.justice.gc.ca/eng/acts/c-6/fulltext.html

Government of Canada. (2020a). Canada gazette, part I, volume 154, number 46: Pay equity regulations. https://gazette.gc.ca/rp-pr/p1/2020/2020-11-14/html/reg1-eng.html

Government of Canada. (2020b). Fertility. https://www.canada.ca/en/publichealth/services/fertility/fertility.html

Kim, D. D., Silver, M. C., Kunst, N., Cohen, J. T., Ollendorf, D. A., \& Neumann, P. J. (2020). Perspective and costing in cost-effectiveness analysis, 1974-2018.

PharmacoEconomics, 38(10).1135-1145.

Lundborg, P., Plug, E., \& Rasmussen, A. W. (2017). Can women have children and a career? IV evidence from IVF treatments. American Economic Review, 107(6).1611-37. 
Mahboob, T.(2020). No national plan for fertility treatment remains challenge for Canadians hoping to conceive. CBC Radio. https://www.cbc.ca/radio/outintheopen/insideinfertility-1.5411669/no-national-plan-for-fertility-treatment-remains-challenge-forcanadians-hoping-to-conceive- 1.5411676

National Assembly of Quebéc. (2020). Projet de loi no 73: An act to amend various provisions relating to assisted procreation. http://m.assnat.qc.ca/fr/travauxparlementaires/projets-loi/projet-loi-73-42-1.html

Orr, S., \& Wolff, J. (2015). Reconciling cost-effectiveness with the rule of rescue: The institutional division of moral labour. Theory and Decision, 78(4). 525-538.

Papazoglou, M. \& Galariotis, I. (2020). Revisiting the effect of income on health in Europe: Evidence from the 8th round of the European social survey. Social Indicators Research, 148(1). 281- 296.

Whitehead, S. J. \& Ali, S. (2010). Health outcomes in economic evaluation: The QALY and utilities. British Medical Bulletin, 96(1). 5-21

World Health Organization. (2017). WHO methods and data sources for global burden of disease estimates 2000-2015 